\title{
Real-time Colorized Video Images Optimization Method in Scotopic Vision
}

\author{
Yong Chen*, Feng Shuai, Di Zhan \\ Key Laboratory of Industrial Internet of Things\& Network Control, MOE, \\ Chongqing University of Posts and Telecommunications, Chongqing, China, 400065 \\ *Corresponding author, e-mail: chenyong@cqupt.edu.cn
}

\begin{abstract}
In low light environment, the surveillance video image has lower contrast, less information and uneven brightness. To solve this problem, this paper puts forward a contrast resolution compensation algorithm based on human visual perception model. It extracts $Y$ component from the YUV video image acquired by camera originally to subtract contrast feature parameters, then makes a proportional integral type contrast resolution compensation for low light pixels in $Y$ component and makes index contrast resolution compensation for high light pixels adaptively to enhance brightness of the video image while maintains the $U$ and $V$ components. Then it compresses the video images and transmits them via internet. Finally, it decodes and displays the video image on the device of intelligent surveillance system. The experimental results show that, the algorithm can effectively improve the contrast resolution of the video image and maintain the color of video image well. It also can meet the real-time requirement of video monitoring.
\end{abstract}

Keywords: video image enhancement, contrast resolution, low-level-light

Copyright $@ 2015$ Institute of Advanced Engineering and Science. All rights reserved.

\section{Introduction}

In recent years, digital image processing technology is widely used in video surveillance, medical testing, image communications and other fields. The quality of video images captured in appropriate illumination scenes will be much better. However, when video surveillance is applied in actual cases, the quality of images is unsatisfactory due to the uncontrollable environment $[1,2]$. The collected images will demonstrate dark and low contrast overall in low illumination, and human eyes can hardly perceive the useful information. Therefore, recovering details of low illumination image, which human eyes can hardly perceive, has practical significance for video surveillance [3].

In AINDANE algorithm proposed by Li et al [4], global contrast enhancement was implemented to improve image quality, but the performance cannot meet the requirement for common applications when dealing with low-contrast images. Tan [5] has improved the image quality by maximizing local contrast based on a physical degradation model. However, the image tends to be saturated and appear halo artifacts after enhancing local contrast merely. The improved histogram equalization algorithm proposed by $\mathrm{Li} \mathrm{CH}$ et al [6] is preferably maintained the color information of the image to some extent, but the enhancement effect is better for a relatively narrow range of gray scale. In [7], a visual compensation method was presented based on the model NR-IQA, combining with the resolution characteristics of the human eyes to compensate the image information, this algorithm shows good performance in low light image scenes, but not for such scene which contains low-light and high-light region or has uneven luminance. Literature [8] developed a Retinex image enhancement algorithm based on Zernike matrix, which has more sensitivity in enhancing the dynamic image at a wider range, but it is intensive computationally and difficult to meet the requirements of real-time video processing. An enhancement algorithm was proposed based on the dark channel priori low light image [9]. This method improves the enhancement effect and reduces the complexity of the algorithm, but it is still hard to adapt to the real-time video processing.

Based on the analyses mentioned above, according to the characteristics of video surveillance in changing low-light environments, the method which combined color image quality evaluation model and image enhancement algorithms was proposed to optimize each dark and 
bright vision pixel based on the human visual characteristics, and using self-optimizing contrast resolution parameter compensation method can avoid the tedious manual settings. Experiments show that this method can effectively enhance the video images captured in low light scene and keep the original image color information more to improve the capability of real-time video processing.

Well, the rest of this paper is organized as follows. In Sect.2, we review the base of image optimizing and evaluation with Sect.3 describing adaptive contrast resolution compensation method. Sect.4 gives the hardware system, and Sect.5 provides a set of test results. The conclusion follows up after this section.

\section{Basic Image Optimization and Evaluation}

\subsection{Contrast Resolution Constraints}

The literature $[10,11]$ studied contrast resolution with the variation of the gray background. For [0,255] grayscale image, means that gray value is between 0 and 47 , and photonic is between 48 and 255. For different optical area, the minimum gradation difference of human vision change along with gray background, but it is non-linear. For the image in scotopic region, part of the target image information which is masked by low-contrast background, can not be identified by the human eyes. Therefore, in this paper, the image target chromaticity level which is difficult to distinguish was just compensated to the extent of the human eyes can distinguish, so as to achieve mining conceal information and improving image quality.

\subsection{Zadeh Transform}

The minimum difference of gray in an image that human visual contrast resolution can distinguish called JND (Just Noticeable Difference) [7]. Human visual contrast resolution nonlinear compensation principle is that expanding a gray level between adjacent pixels to one JND, then useful information is masked by the background so that they can be resolved to the extent that can be distinguished.

\subsection{Color Image Quality Evaluation Index}

Human vision problems are psychological physical problems; the image quality is also a psychological evaluation of physical problems [12]. Quoting comprehensive color image quality evaluation function expression (1) from $[13,14]$.

$$
\mathrm{CAF}=\mathrm{AIE} \times \mathrm{APCL} \times \mathrm{NNF} \times \mathrm{AHF} \times \mathrm{ABWF}
$$

Where AIE, APCL, AHF, NNF and ABWF represent the information entropy, the physical contrast, average level factor, the average brightness normalization are close to distance and average bandwidth factor respectively. The bigger the CAF value is, the better the image quality of human visual perception will be.

\section{Adaptive Contrast Resolution Compensation Method \\ 3.1. Video Image Contrast Resolution Compensation}

The format of video image collected by the front-end camera is usually $Y U V$, where $Y$ is the luminance or brightness, and $U$ and $V$ are the $R-Y$ and $B-Y$ component respectively, also known as chroma which describes color saturation attribute [15]. The advantage of $Y U V$ is that luminance signal $(Y)$ and chrominance signal $(U, V)$ are mutual independent and two components $U$ and $V$ can represent color which means compression, transmission and processing are more easier. Making use of those YUV features, a compensation method is proposed to optimize $\mathrm{Y}$ component without losing color information of images while the $\mathrm{U}$ and $\mathrm{V}$ are constants.

Based on characteristics of human vision contrast resolution limit, it combines the JND with proportional integral operation of automatic closed-loop control system to optimize the pixel whose gray scale value range from 0 to 47 (in scotopic region) using proportional-integral-type contrast resolution compensation optimization method. The equation is shown as Equation (2). 


$$
T(x, y, Y)=k \sum_{i=0}^{i-O G(x, y, Y)} J N D(i)
$$

Where $T(x, y, Y)$ represents the target luminance value of the compensated dark vision pixels in $Y$ component image, $O G(x, y, Y)$ represents the original luminance of scotopic pixel in $Y$ component image. $\mathrm{k}$ represents the compensation coefficient which is a positive real number, and JND (i) represents Just Noticeable brightness distance on the background brightness i.

If only compensate $Y$ component of the dark vision pixel, image layering will be reduced, image information will be imbalance and the image will be over enhanced partially. Therefore, the $Y$ component of photopic vision also needs to be optimized and compensated. Just noticeable resolution of clear visual is in range at 1.17-1.75 [6]. Based on human visual has a certain adaptive characteristics to visual images, combined the global mapping method, more in line with the human eye for high dynamic image identification, with exactly resolvable JND. According to Equation (2), establish exponential contrast resolution compensation optimization method under clear visual as Equation (3) shows.

$$
T(x, y, Y)=O G(x, y, Y) \times\left(\sum_{i=0}^{i-O G(x, y, Y)} J N D(\mathrm{i})\right)^{r}
$$

Where $\mathrm{T}(\mathrm{x}, \mathrm{y}, \mathrm{Y})$ represents the target luminance value of the compensated darkvision pixels of the $\mathrm{Y}$ component image, $\mathrm{OG}(\mathrm{x}, \mathrm{y}, \mathrm{Y})$ represents the original luminance of scotopic pixel of the $Y$ component image, $r$ represents a variable parameter adjusting compensation depth, and JND (i) represents exactly resolvable brightness distance on the background brightness i. For the image, the average brightness is in dark vision area, When the pixel luminance value of the $Y$ component image less than or equal 47, we use Equation (2) to carry out proportional-integral-type contrast resolution compensation under the dark vision. When the pixel brightness values of the $Y$ component image greater than 47 and less than equal to 255 , we use Equation (3) to carry out proportional-integral-type contrast resolution compensation under the clear vision. According to the above, contrast resolution compensation was proposed for color image, $\mathrm{CRCCl}$ as Equation (4) shows.

$$
T(x, y, Y)=\left\{\begin{array}{l}
O G(x, y, Y) O G(x, y, Y)=0 \\
k \sum_{i=0}^{i=O G(x, y, Y)} J N D(i) \quad O G(x, y, Y) \leq 47 \\
O G(x, y, Y) \times\left(\sum_{i=0}^{i=O G(x, y, Y)} J N D(i)\right)^{r} 47<O G(x, y, Y) \leq 255
\end{array}\right.
$$

To avoid the phenomenon of anti-color complementary color that original brighter place will be dimmed and deformity after compensation optimized. For the pixels that compensated target brightness values greater than 255, set the value to 255 , and If the value is less than 0 , set the value to 0 . Equation (5) shows the constraints:

$$
T(x, y, Y)=\left\{\begin{array}{l}
0, T(x, y, Y)<0 \\
255, T(x, y, Y)>255
\end{array}\right.
$$

Due to the uncontrollable nature of lighting conditions in video surveillance and the continuity of the video [16], to make each frame to achieve optimum adaptive compensation effect, select the image mean variance which can be better characterize image parameters as the adaptive parameter. Equation (6) is a mean square error characterizing the image feature, Where $\mathrm{K}(\mathrm{i}, \mathrm{j})$ is the image average gray. 


$$
V \mathrm{ar}=\sqrt{\frac{1}{M \times N} \sum_{I-0}^{M-1} \sum_{j-0}^{N-1}\|I(i, j)-K(i, j)\|^{2}}
$$

\subsection{Adaptive Contrast Resolution Parameter Selection}

Through experiments respectively build the relationships between $\mathrm{k}$ (compensation coefficient) and mean square error under dark vision, the relationship between $\mathrm{R}$ (compensation coefficient) and the mean square under clear vision. Select a picture with a low-light environment as experimental material from the Color Checker Dataset Gallery (http://www.eecs.harvard.edu/ ayanc/oldcc/dbs.html). By constantly disturbing the compensation coefficient $k$ and $r$ to look for the maximum value of the objective parameters (CAF) representing the image quality, and combined with subjective evaluation to determine the best quality image after optimization. As shown by the curve fitting, Figure 1 and Figure 2 show this relationship.

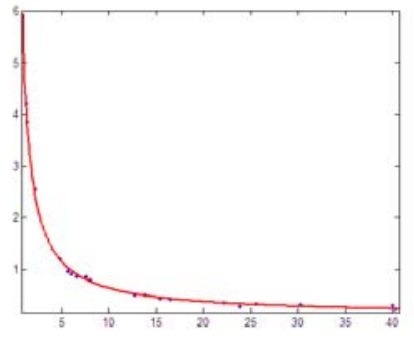

Figure 1. Relationship between $\mathrm{k}$ and mean square deviation in scotopic vision

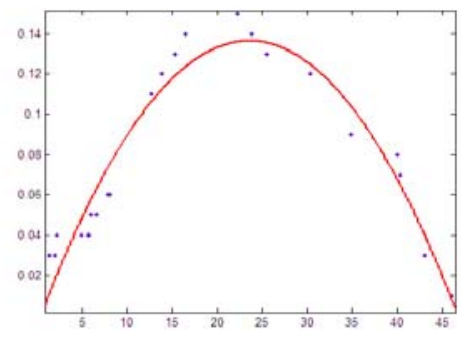

Figure 2. Relationship between $r$ and mean square and mean square error in photonic vision

Thereby establishing a function, as Equation (7) shows, between compensation coefficient $\mathrm{k}$ and the mean square error of the original image under dark vision conditions.

$$
k=5.138 / \operatorname{Var}+0.113
$$

The same method can be used to performed $r$ index compensation coefficient automatic optimization on the original image under clear vision conditions. Create coefficient $r$ and the mean square error of the original image as Equation (8) shows.

$$
r=-2.4645 \times 10^{-4} \operatorname{Var}^{2}+0.0117 \operatorname{Var}-0.0308
$$

\subsection{Adaptive Contrast Resolution Compensation of the Video Image}

First, calculate mean square error for each frame of video image captured from the front camera. Then, calculate $k, r$ value of each frame image by the mean square error; optimize the $Y$ component of each frame image by adaptive compensation optimization. Holding $U, V$ components of the video stream are for the same to give a final enhanced video image of YUV space. Finally, convert the enhanced video image into an RGB color image and show it in the monitoring equipment.

\section{Hardware system}

As Figure 3 shows, the system comprises a processing (DM6467) unit and a video input units. 


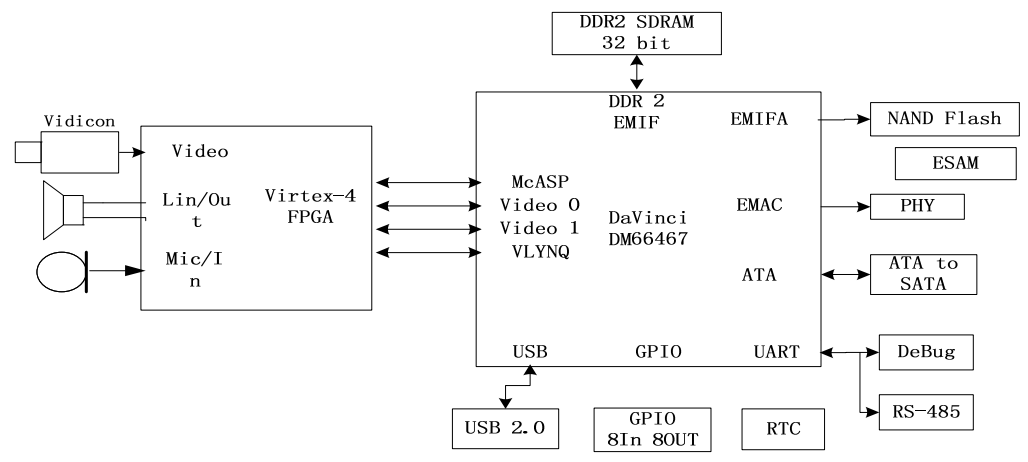

Figure 3. Hardware system diagram

\subsection{The Experimental Results and Subjective Evaluation}

The TM320DM6467T was used as main processor chip of the core of the system processing unit, the chip integrates a high-performance TMS320C64x + DSP core and an ARM926EJ-S core. And the clock frequency reaches $1 \mathrm{GHz}$, in which DSP is mainly responsible for compression and related video data algorithm, and ARM is primarily responsible for communication with peripherals and related scheduling.

\subsection{Video Input and Output Unit}

Video capture module consists of a CCD camera and a Xilinx Virtex-4 FPGA processor. The video stream captured by acquisition module is passed to the FPGA processor by TVP5150, and FPGA will complete the switching among interfaces $\mathrm{YCbCr} / \mathrm{HDMI} / \mathrm{HDSDI} /$ VGA. To improve the collection efficiency, use time-multiplexing to ensure multi-channel signal input. Transport data to DDR2 for the use of DM6467T through DMA mode.

\section{Integrated Experiment}

Software design of the system is based on an embedded Linux operating system application software platform, including video capture, video pre-processing, video processing algorithms and compression, network transmission, the client decodes display module.

The experimental system captures video stream with CCD camera equipment and follows Video4Linux2 (referred V4L2) frame flow, takes out the original video stream in the frame buffer through getCaptureBuffer(), calculates the overall average variance for each frame image, the $k, r$ values are obtained by calculating the average variance of the frame image, and extracts the $Y$ component to make adaptive compensation, maintains the $U, V$ components of the video stream for the same, then the enhanced image is obtained in the YUV space. Call codec of DSP side by Codec Engine mechanism, call H.264 encoder for encoding the processed video stream, then the data was transmitted through RTP network protocol and displayed after decoding in monitoring terminal.

Specific steps: First, initialize the engine through calling the initialization module CERuntime_init () of the core engine API; Then open the encoding engine and return a hEngine pointer through calling Engine_open (); Followed, create a coded handle calls by Venc1_create () through calling hEngine pointer; At last through calling Venc1_process () to achieve the video stream encoding by H.264 encoder, then the encoded video stream is transmitted over the network and then displayed in the client after decoder decoding. Experimental design flow chart is shown in Figure 4. 


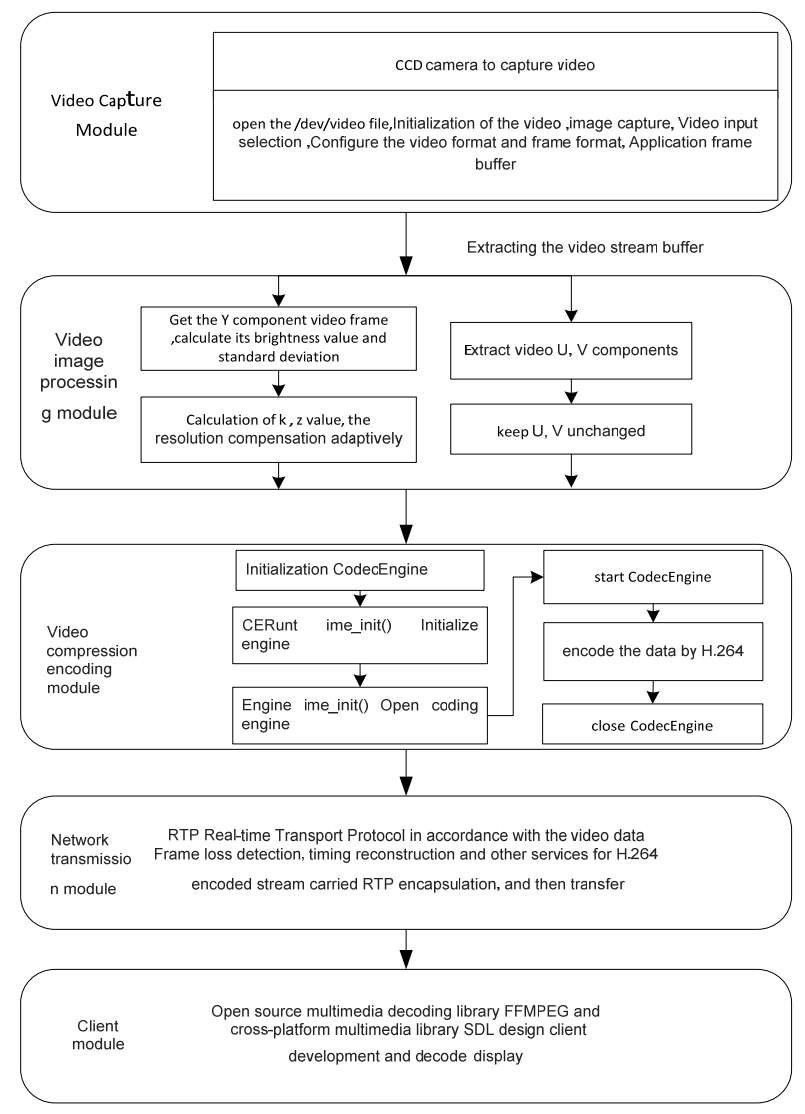

Figure 4. Experiment design flow chart

\subsection{Analysis of Experimental Results}

The improved bidirectional histogram (abbreviated: I-HE) [17], an improved multi-scale Retinex (abbreviation: I-MSR) [18-20] and contrast resolution compensation method (referred to as: $\mathrm{CRC}$ ) were used to compare. The experimental environment includes three kinds of video images (video surveillance sites: http://www.vcn.com.cn/) and a set of video with different light, different traffic monitoring sites.

\section{1) Subjective evaluation}

Experiment one: Figure 5(a) shows a video under low-lighting traffic conditions. The chroma spectra analysis shows that this video frame chroma level is relatively concentrated, low contrast, narrow bandwidth, useful information is difficult to be identified by the human eyes. Figure 5(b) shows an image which was processed by improved bidirectional histogram equalization. The entire image chroma level has been stretched, but the tensile range of high density chrominance level is larger than the range of low-density chrominance level and there has been the image contrast stretching unevenness, resulting in color abnormal and severe color distortion. Figure 5(c) shows the image processed by multi-scale Retinex enhancement method, according to the optimization effect, chroma level image has been effectively stretched, and details of the image are highlighted. But a lot of chroma spectral are between $100-160$, which make the overall image too bright and appears whitish phenomenon, the vehicle operating conditions is difficult to identify in the figure. Figure 5(d) shows the image which was processed by contrast resolution compensation, image layering processed by this method is significantly stronger, chroma spectral distribution is more uniform, and the color of the image has been well maintained, the color proportion of the vehicle and the road surface becomes coordination to facilitate real-time monitoring. 


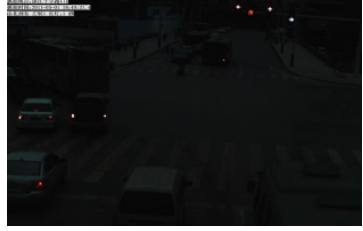

(a) Original image

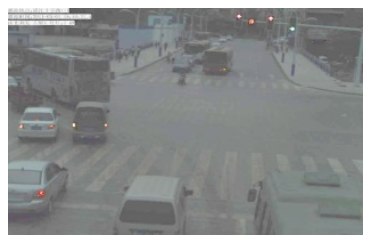

(c) Improved Multi-scale Retinex
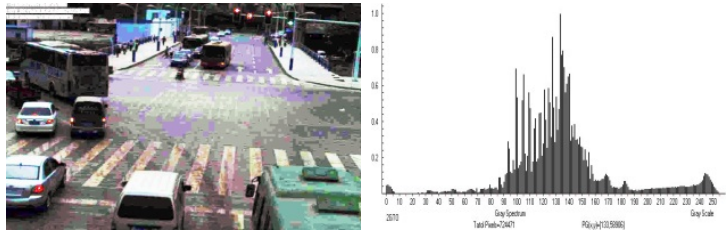

(b) Improved two-dimensional histogram
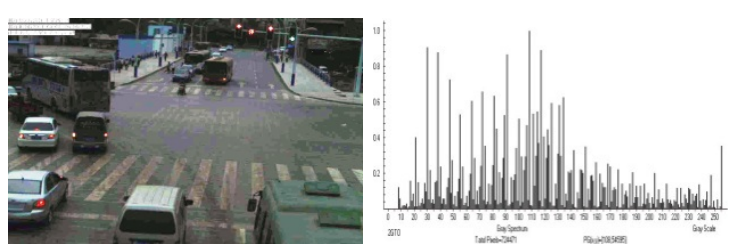

(d) Contrast Resolution Compensation

Figure 5. Traffic record and 2 level chroma spectra

Experiment two: For uneven illumination appeared in video surveillance, such as the case of point light source in Figure 6 , a partial region of the image appears in strong light, and a partial region of the image appears in low light. Process the image through improved bidirectional histogram, although the brightness of the entire image was enhanced, however, the lighter areas of the processed image occurred the effort of over enhancement, and the lower illumination region enhanced obviously, a color distortion occurs. The image which was improved by Multi-scale Retinex processing was shown in the Figure 6(c). The overall image occurred over enhanced, the picture appeared whitish and the stations was more difficult to identify. Figure 6(d) shows the image after contrast resolution compensation processing, chroma spectral distribution is more uniform, maintains good color, and less detail is lost, more in line with the human eye.

Experiment three: the original image in Figure 7-8 was with uneven illumination and the lower overall image brightness, after optimized by the improved bidirectional histogram the image shown in Figure 7(b), 8(b) appeared uneven distribution of light and dark, pavement color imbalance caused poor overall visual effect of the image. The image which was processed by improved Multi-scale Retinex processing was shown in Figure 7(c) and 8(c), the overall image occurred over enhancement, more image information is lost. The images which were processed by contrast resolution compensation shown in Figure 7(d) and 8(d), image information is good, information is evenly distributed, color information well maintained, and pavement color uniformity is more suitable for the human eye to observe.

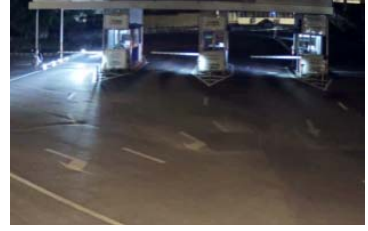

(a) Original image

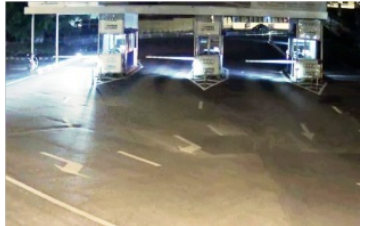

(b) Improved twodimensional histogram

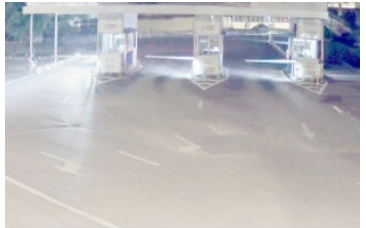

(c) Improved Multiscale Retinex

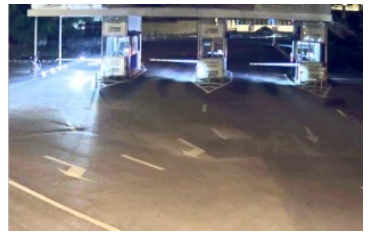

(d) Contrast Resolution Compensation

Figure 6. Gas station effect 


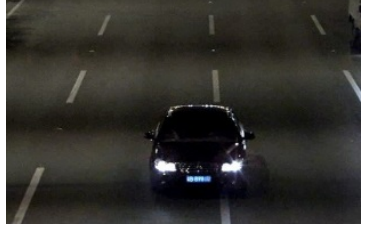

(a) Original image

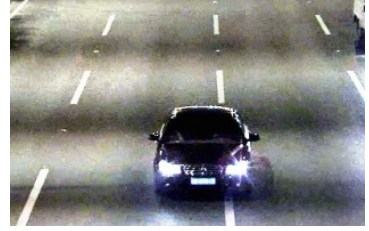

(b) Improved twodimensional histogram

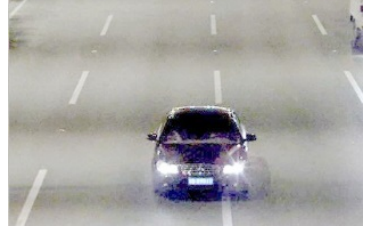

(c) Improved

Multiscale Retinex

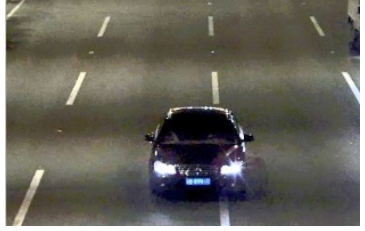

(d) Contrast

Resolution

Compensation

Figure 7. High speed car effect

\section{2) Objective evaluation}

Select information entropy, contrast, and CAF objective indicators to calculate three groups of video image and a set of video, after processed by two-way histogram, improved multi-scale Retinex and contrast resolution compensation methods. The values are shown in Table 1, Table 2. After using contrast resolution compensation, image information entropy and contrast as well as the CAF value are significantly larger than other methods, which shows the consistency of subjective and objective evaluation, and indicates that the video which processed by the proposed treatment methods has better quality.

Table 1. Video image quality assessment

\begin{tabular}{ccccc}
\hline $\begin{array}{l}\text { Method } \\
\text { arameter }\end{array}$ & & Information Entropy & Contrast & CAF \\
\hline \multirow{2}{*}{ Fig.5 } & I-HE & 5.136 & 1.145 & 5.125 \\
& I-MSR & 5.867 & 0.766 & 4.367 \\
& CRC & 7.577 & 2.541 & 19.146 \\
& I-HE & 6.956 & 2.339 & 8.584 \\
Fig.6 & I-MSR & 6.835 & 1.587 & 5.043 \\
& CRC & 7.322 & 2.403 & 15.099 \\
& I-HE & 6.088 & 2.231 & 5.400 \\
Fig.7 & I-MSR & 6.362 & 1.970 & 6.569 \\
& CRC & 6.449 & 2.971 & 10.324 \\
\hline
\end{tabular}

\section{3) Real-time verification}

To validate the real time of the algorithm, Figure 8 is the 100 frames of video traffic monitoring. With respect to the improved bidirectional histogram equalization (Figure 8(b)), the effect of the image processed by the improved multi-scale Retinex algorithm (Figure 8(c)) is more significant. However, this method uses linear iterative manner to perform the time-domain filtering for the illumination component of the image which has large amount of computation. The average time of processing a surveillance video with the improved multi-scale Retinex algorithm is $0.18 \mathrm{~s}$, and the frame rate is about 5 frames $/ \mathrm{sec}$. Bidirectional histogram processing has faster speed, but it also needs to count video frame pixel probability distribution and do it twice, so the average time of processing each frame takes $92 \mathrm{~ms}$, a frame rate of 10 frames/sec. Contrast resolution compensation, due to the compensation optimization method is relatively simple, the time complexity of the algorithm is low, the average time of processing each frame is $48 \mathrm{~ms}$, frame rate is $20 \mathrm{frames} / \mathrm{sec}$, which is significantly better than the other two methods in order to meet the requirements of real-time in video processing as shown in Table 2. 


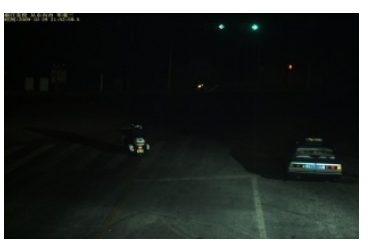

(a) Original image

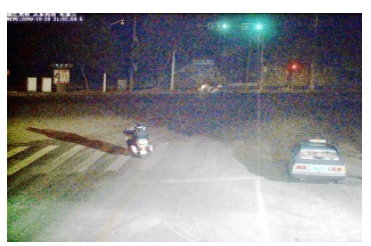

(b)Improved twodimensional histogram

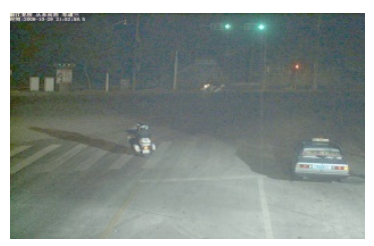

(c) Improved Multiscale Retinex

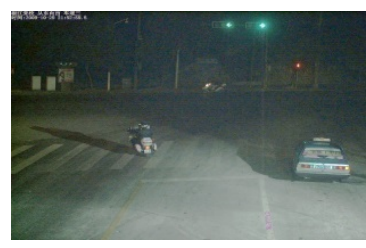

(d) Contrast Resolution Compensation

Figure 8. Traffic light intersection effect

Table 2. Real-time video image evaluation parameters

\begin{tabular}{llrrcc}
\hline Method Parameter & $\begin{array}{c}\text { Information } \\
\text { Entropy }\end{array}$ & Contrast & CAF & Frame rate (frames / sec) \\
\hline \multirow{2}{*}{ Fig 8 } & I-HE & 5.245 & 2.396 & 1.879 & 10 \\
& I-MSR & 7.075 & 2.358 & 11.145 & 5 frames $/ \mathrm{sec}$ \\
& CRC & $\mathbf{7 . 1 8 2}$ & $\mathbf{2 . 4 3 8}$ & $\mathbf{1 5 . 8 0 0}$ & $\mathbf{2 0}$ frames / sec \\
\hline
\end{tabular}

\section{Conclusion}

Based on the human visual characteristic, under the low illumination, process the pixels in different areas of the video image through different types of contrast resolution compensation. And through self-optimizing optimal compensation parameters, maintain $\mathrm{U}, \mathrm{V}$ component unchanged, so that the brightness of the image has been enhanced to ensure the image color saturation. Finally by coding transmission network, the image is decoded and displayed in the client and form a complete monitoring system. Through the analysis of subjective and objective evaluation, The proposed algorithm in the overall enhancement of the video image, color maintaining and light recovery have achieved good results. And the average time of this algorithm processing video is $48 \mathrm{~ms}$, which complies with the requirements of realtime video surveillance to ensure the overall effect of the video.

\section{Acknowledgements}

Authors would like to thank the Chongqing Education Committee Science of China for supporting the Foundation of program, No KJ1400434.

\section{References}

[1] CHEN D, TIAN F C, LIU Y, HU Y W, HAN L. Image Enhancement in Coherent Optical Amplification by Photorefractive Crystals. Journal of Computer and Communications. 2014; 2: $42-47$.

[2] NAYAK DR, BHOI A. Image Enhancement Using Fuzzy Morphology. Journal of Engineering Computers \& Applied Sciences. 2014; 3(3): 22-26.

[3] Bennett EP, McMillan L. Video enhancement using per-pixel virtual exposures. ACM Transitions on Graphics(TOG). 2005; 24(3): 845-852.

[4] LI T, ASARI V. An integrated neighborhood dependent approach for nonlinear enhancement of color images. Proceedings of the IEEE Computer Society International Conference on Information Technology: Coding and Computing-ITCC. 2004; 2: 138-139.

[5] TAN K, OAKLEY PJ. Physics-based approach to color image enhancement in poor visibility conditions. Optical Society of America. 2001; 18(10): 2460-2467.

[6] $\mathrm{LI} \mathrm{CH}$, LIU H, ZHONG C. Application of histogram modification in image enhancing. J. Hubei University of Techno. 2011; 26(2): 67-70.

[7] Stark JA. Adaptive image contrast enhancement using generalizations of histogram equalization. IEEE Trans. Image Processing. 2000; 9(5): 889-896.

[8] CHEN Y, CHENG JJ, XIE ZHX. A compensation method of human visual system based - on NR-IQA. Journal of Chongqing University. 2013; 36(2): 141-147.

[9] WANG RG, ZHANG XT, ZHANG X, FANG S. A novel Retinex algorithm for image enhancement based on Zernike moment. Journal of Image and Graphics. 2011; 3(16): 310-315. 
[10] Land EH. Recent advances in Retinex theory and some in placations for contical computations color vision and the natural image. Proceeding of National Academy of Sciences. 1983; 80(16): 5163-5169.

[11] DI XG, QU YY. An improved low illumination image enhancement algorithm with color preserving. Journal of Harbin Institute of Technology. 2014; 46(3): 1-7.

[12] FAN YY, SHEN XH, SANG YJ. No reference image sharpness assessment based on contrast sensitivity. Opt. Precision Eng. 2011; 19(10): 2485-2493.

[13] XIE ZHX, LIU YH, WANG ZHF, XIONG XL, HU Q. A nonlinearly compensatory principle and method for human vision contrast resolution. Journal of Chengdu Medical College. 2009; 4(3): 157-162.

[14] CHEN Y, LI Y, Lü XF, XIE ZX, FENG P. Active assessment of color image quality based on visual perception. Optics and Precision Engineering. 2013; 21(3): 2534-2542.

[15] MA GF, YANG JH, ZHOU B. Motion detection in video based on YUV color space. Computer Engineering and Design. 2008; 29(14): 3700-3708.

[16] MA YD, TENG F, ZHAN K, ZHANG HJ. A New Method of Color Image Enhancement Using Spiking Cortical Model. Journal of Beijing University of Posts and Telecommunications. 2012; 35(3): 70-73.

[17] ZHAO XX, WANG RL, ZHANG LL. Research of enhancement algorithm for degraded images in adverse weather. Microcomputer Information. 2010; 26(8): 3-5.

[18] LEE CH, SHI JL, LIEN CHCH, HAN CHCH. Adaptive Multiscale Retinex for Image Contrast Enhancement. Signal-Image Technology \& Internet-Based Systems, 2013 International Conference on IEEE. 2013: 43-50.

[19] Lu Y, Guo L, Li H. Remote sensing image fusion edge information and features of SAR image based on curvelet transform. Acta Photonica Sinica. 2012; 41(9): 1118-1123.

[20] Yong Chen, Jie Xiong, Huan-lin Liu, Qiang Fan. Combine Target Extraction and Enhancement Methods to Fuse Infrared and LLL Images. TELKOMNIKA Indonesian Journal of Telecommunication, Computing, Electronics and Control). 2014, 12(3): 605-612.

[21] Jianhua $L$, Jianguo $\mathrm{Y}$. Multifocus image fusion by SML in the shearlet subbands. TELKOMNIKA Indonesian Journal of Electrical Engineering. 2014; 12(1): 618-626. 